\title{
Trastorno de ansiedad por separación
}

\author{
BERNARDO PACHECO P. ${ }^{1}$, TAMARA VENTURA W. ${ }^{2}$ \\ 1. Psiquiatra Infanto Juvenil. Departamento de Psiquiátria, Facultad de Medicina, Pontificia Universidad Católica de Chile. \\ 2. Médico Cirujano, Pontificia Universidad Católica de Chile.
}

\begin{abstract}
Separation anxiety disorder and developmental separation anxiety

Anxiety disorders constitute the most common psychiatric diseases in children. These disorders predict an increase in the risk of later anxiety disorders, mayor depression and hospitalization for psychiatric pathology. The primary feature of Separation Anxiety Disorder is developmentally inappropriate anxiety sufficient to cause clinically significant distress or impairment when faced with separation from home or major attachment figures. Drugs that selectively inhibit serotonin reuptake, psychotherapy and parent education constitute effective treatments for children and adolescents with anxiety disorders. Pediatricians and other health care professionals are in a unique position to assist families in understanding the etiology, prevention and treatment of separation anxiety disorder. Early identification in pediatric primary care and effective management may help improve outcome. The objective of our review is to analyze clinical and therapeutic aspects of the Separation Anxiety Disorder and its differences with Separation Anxiety as an expression of the evolutionary emotional development in children.

(Key words: Anxiety disorders, separation childhood).

Rev Chil Pediatr 2009; 80 (2): 109-119
\end{abstract}

\section{RESUMEN}

Los trastornos por ansiedad son cuadros clínicos psiquiátricos frecuentes y predicen un riesgo aumentado de presentar posteriormente trastornos ansiosos y depresivos, además de hospitalizaciones por causa psiquiátrica. El trastorno por ansiedad de separación tiene como característica principal ansiedad excesiva e inapropiada para el desarrollo del niño, concerniente a su separación respecto de las personas con quienes está vinculado. El uso de fármacos, psicoterapia y apoyo parental son medidas terapéuticas eficaces. Los pediatras pueden ser los primeros profesionales que tomen contacto clínico con los niños y realicen la primera aproximación diagnóstica y terapéutica. El propósito de nuestra revisión es analizar aspectos clínicos y terapéuticos del trastorno de ansiedad por separación y sus diferencias con la ansiedad de separación propia del desarrollo emocional del niño.

(Palabras clave: ansiedad por separación, trastorno ansioso, niños).

Rev Chil Pediatr 2009; 80 (2): 109-119

Trabajo recibido el 16 de octubre de 2008, devuelto para corregir el 22 de diciembre de 2008, segunda versión el 12 de enero de 2009, aceptado para publicación el 26 de enero 2009.

Correspondencia a:

Dr. Bernardo Pacheco P.

E-mail: bejupa@vtr.net 


\section{Introducción}

La ansiedad por separación (AS) es una respuesta emocional en la cual el niño experimenta angustia al separarse físicamente de la persona con quién está vinculado (generalmente su madre). La AS es un fenómeno normal, esperable y obligado del desarrollo infantil, que comienza a manifestarse alrededor de los 6 u 8 meses de edad ${ }^{1}$. La AS permite que el niño desarrolle paulatinamente su capacidad de estar a solas. Contribuye activa y necesariamente a este proceso la figura de apego que el niño dispone.

El trastorno de ansiedad por separación (TAS) es una manifestación psicopatológica, caracterizada por la incapacidad del niño de quedarse y estar a solas. En este caso el niño no es capaz de separarse apropiadamente de la persona que es emocionalmente significativa para él. La angustia del TAS es excesiva y sobre lo esperado para el nivel de desarrollo del niño, es decir, no es una angustia normativa. El diagnóstico clínico de un TAS puede ser difícil de realizar, ya que muchas veces no es fácil diferenciar formas normativas de formas patológicas de ansiedad por separación. Nuestro propósito en éste artículo es revisar las diferencias clínicas existentes entre la AS como expresión del desarrollo emocional evolutivo y el TAS como expresión psicopatológica. Además analizaremos aspectos diagnósticos y terapéuticos del TAS.

\section{Conceptos generales respecto a la angustia}

La ansiedad como emoción es parte constitutiva del desarrollo humano. Puede experimentarse como incomodidad emocional asociada a la anticipación de peligro o de amenaza. La ansiedad es un estado de espera expectante y no siempre tiene connotación negativa. La ansiedad está presente a lo largo del desarrollo en tareas que el individuo debe resolver en el proceso de construcción de su persona. Se ha usado el término angustia para referirse al correlato físico de la ansiedad, es decir su expresión corporal. Para nuestro fin los términos ansiedad y angustia los usaremos como sinónimos. Los miedos o temores se asemejan a la ansiedad en ser emociones básicas, pero se diferencian en que las primeras generalmente se relacionan con una situación u objeto que es temido e identificado por el sujeto. Entendemos por ansiedad normal aquella respuesta emocional al entorno que es adecuada al nivel de desarrollo evolutivo y que promueve en el sujeto una respuesta de adaptación al medio. La ansiedad patológica puede ser conceptualizada como aquel estado de expectación o preocupación excesiva que interfiere con las actividades normales de la persona y cuya expresión no es proporcional al nivel de desarrollo del individuo $^{2}$. Ya que existe una ansiedad normativa en respuesta a conflictos del desarrollo psicológico, no debe entenderse a la ansiedad en sí misma como un estado mental problemático o disfuncional que debiera ser erradicado.

Desde la perspectiva psicoanalítica, la ansiedad se conecta al superyó (las normas, la conciencia de lo correcto) y se manifiesta como un sentimiento de preocupación, de culpa o pena de no poder cumplir con un ideal interno de comportamiento moral $^{3}$. En los estadios tempranos del desarrollo infantil, la ansiedad se asocia al temor de perder el afecto o la aprobación de los progenitores. Una fuente de angustia crucial en el desarrollo temprano, es experimentar la posibilidad de perder no sólo el afecto del objeto amado (el cariño de la madre) sino perder el objeto amado en sí mismo (perder a la madre). Este estado emocional se expresa como ansiedad de separación. Desde una perspectiva más relacional, es decir en el contexto de la relación del sujeto con sus seres queridos, muchos niños y adolescentes e incluso adultos pueden experimentar cierto grado de AS al encontrarse distanciados de las personas o ambientes que les son familiares. El proceso de diferenciación que cada miembro familiar debe hacer de su propia familia para alcanzar mayor autonomía emocional, puede ser experimentado con angustia a la separación del grupo $^{4}$.

\section{Aspectos epidemiológicos del TAS}

El TAS es uno de los trastornos de ansiedad más común en los niños. Los reportes de prevalencia en varios estudios van desde un $2,4 \%$ a $5,4 \% 5$. La prevalencia de trastornos con sintomatología ansiosa es mucho mayor pudiendo 
llegar hasta al 50\% en niños. En muestras epidemiológicas se ha observado que el TAS tiende a ser más frecuente en mujeres, aunque otros estudios no han demostrado diferencias por género, sí han encontrado que los niños reportan mayor número de síntomas ansiosos comparados con los adolescentes ${ }^{6}$. La mayor frecuencia del TAS surge en la infancia media entre los 7 y 9 años de edad, aunque puede ser más temprano, incluso durante la etapa preescolar. Aunque se conoce que la prevalencia del TAS disminuye con la edad, la ansiedad o conductas de evitación relacionadas a la separación, pueden persistir hasta la adultez ${ }^{7}$. Los niños o adolescentes que presentan un TAS cuando sean adultos tendrán mayor predisposición a presentar trastornos de angustia con agorafobia $^{8}$.

\section{Diagnóstico y características clínicas}

Para el clínico puede ser difícil detectar y cuantificar la ansiedad excesiva en sus pacientes. Dado que los niños ansiosos y sus padres con frecuencia consultan primero al pediatra antes que al profesional de la salud mental, éste debe estar preparado para incorporar en su evaluación estrategias de entrevista psicológica.

La ansiedad excesiva relacionada a la separación puede manifestarse en el paciente como: preocupación, miedo, nerviosismo, tensión o rabia al estar separados del ser querido. En términos corporales la ansiedad se expresa frecuentemente como quejas somáticas; dolores abdominales y cefaleas. Es conveniente tomar la historia clínica tomando como fuente de información al paciente, a sus padres u otros cercanos. Es necesario evaluar la presencia de trastornos psiquiátricos ansiosos y/o depresivos en los padres del niño, así conoceremos la tendencia familiar a la expresión individual de estos trastornos. Observemos (in vivo) en la evaluación del paciente su expresión de ansiedad a la separación de las personas que él quiere o con quienes está vinculado y estimemos su carácter funcional. De este modo podemos obtener una apreciación de la magnitud y el carácter de la AS si la hay.

El TAS está definido en el Manual Estadístico de los Trastornos Mentales (DSM-IV) ${ }^{9}$ como la ansiedad excesiva e inapropiada para la eta- pa del desarrollo del niño en relación a la separación de éste de sus figuras de apego (tabla 1). Las manifestaciones incluyen malestar emocional excesivo y recurrente y/o quejas somáticas repetidas al anticipar el momento de la separación de los cuidadores o del alejamiento del hogar. La somatización o síntoma físico que se da en los niños con TAS deben alertar al clínico a no descartar a priori una enfermedad física contemporánea. Los niños con ansiedad excesiva se rehúsan a estar solos, temen dormir sin compañía y buscan dormir con sus padres. Son reacios a pernoctar fuera de casa y frecuentemente tienen pesadillas de ser abandonados. La negativa de asistir al colegio es un problema común en estos niños. En el colegio intentan llamar al hogar para chequear el paradero y el bienestar de sus padres o repetidamente van a la enfermería con alguna molestia, deseando ser enviados a su hogar y reencontrarse con su figura de apego.

Los niños con TAS son descritos como inseguros, rabiosos y con una necesidad constante de atención. Estas conductas frustran a los padres provocando en ocasiones resentimiento y conflicto que puede traspasarse al interior de la familia ${ }^{10}$. Las manifestaciones del trastorno varían según la edad. Los niños más pequeños exteriorizan el temor más corporalmente y los mayores verbalizan la ansiedad o el temor a peligros potenciales (secuestro, robos, asaltos). Las quejas somáticas como dolor abdominal, cefalea, náuseas e incluso vómitos, pueden presentarse antes y durante la separación de la figura vinculada. Los síntomas cardiovasculares como precordalgia o palpitaciones son más comunes de ser percibidos en los niños mayores $^{10}$.

El TAS puede tener un inicio agudo o insidioso. Frecuentemente es de inicio agudo cuando está precedido por un estrés significativo. Ejemplos de esto son la enfermedad o el fallecimiento de un ser querido como puede ser una mascota. Un cambio de barrio, colegio o ciudad también puede ser un gatillante para el inicio síntomas. El curso del TAS se caracteriza por períodos de exacerbación y remisión, algunos niños se recuperan completamente sin ninguna secuela aparente y otros tienen largos períodos asintomáticos intercalados con períodos de exa- 
Tabla 1. Criterios según DSM IV para el diagnóstico de trastorno de ansiedad por separación (TAS)

A. Ansiedad excesiva e inapropiada para el desarrollo del sujeto, concerniente a su separación respecto del hogar o de las personas con quienes está vinculado, puesta de manifiesto por tres (o más) de las siguientes circunstancias:

(1) malestar excesivo recurrente cuando ocurre o se anticipa una separación respecto del hogar o de las principales figuras vinculadas.

(2) preocupación excesiva y persistente por la posible pérdida de las principales figuras vinculadas o a que éstas sufran un posible daño.

(3) preocupación excesiva y persistente por la posibilidad de que un acontecimiento adverso dé lugar a la separación de una figura vinculada importante porque se produzca un acontecimiento que le separe de las figuras con las que mantiene más vínculos (p. Ej. extraviarse o ser secuestrado).

(4) resistencia o negativa persistente a ir al colegio o a cualquier otro sitio por miedo a la separación.

(5) resistencia o miedo persistente o excesivo a estar en casa solo o sin las principales figuras vinculadas, o sin adultos significativos en otros lugares.

(6) negativa o resistencia persistente a ir a dormir sin tener cerca una figura vinculada importante o a ir a dormir fuera de casa.

(7) pesadillas repetidas con tema de separación.

(8) quejas repetidas de síntomas físicos (como cefaleas, dolores abdominales, náuseas o vómitos) cuando ocurre o se anticipa la separación respecto de figuras importantes de vinculación.

B. La duración del trastorno es de por lo menos 4 semanas.

C. El inicio se produce antes de los 18 años de edad.

D. La alteración provoca malestar clínicamente significativo o deterioro social, académico (laboral) o de otras áreas importantes de la actividad del individuo.

E. La alteración no ocurre exclusivamente en el transcurso de un trastorno generalizado del desarrollo, esquizofrenia u otro trastorno postpsicótico, y en adolescentes y adultos no se explica mejor por la presencia de un trastorno de angustia con agarofobia.

Especificar si:

Inicio temprano: si el inicio tiene lugar antes de los 6 años de edad.

American Psychiatric Association. Manual Diagnóstico y Estadístico de los Trastornos Mentales $4^{\circ}$ ed. (DSM IV). Ed. Masson, Barcelona 1995.

cerbaciones o recurrencias repentinas que pueden ocurrir en momento de mayor exigencia de autonomía. Los trastornos ansiosos provocan un impacto negativo en diversas áreas del funcionamiento del niño, afectando su integración social, su rendimiento académico y alterando su autoestima $^{11}$.

\section{Diagnóstico diferencial}

Ya que la ansiedad excesiva es base común para otros trastornos ansiosos, es necesario distinguir correctamente la ansiedad y sus distintas formas de presentación psicopatológica. Dentro de los diagnósticos diferenciales lo primero es distinguir el TAS de la AS normativa. A modo de orientación clínica los autores muestran en la tabla 2 una comparación entre estas dos condiciones, distinguiendo factores de implicancia clínica que pueden ayudar al diagnóstico.

En el trastorno de ansiedad generalizada, otro trastorno por ansiedad que muchas veces se confunde con el TAS, la ansiedad es excesiva y constante y no aparece sólo ante el alejamiento de la figura vinculada, sino que también en otros temas de preocupación y temor para el niño como es su rendimiento escolar, la salud de sus padres con pensamientos recurrentes sobre la muerte de estos. En las crisis de angustia o pánico el temor en el niño se liga a la ansiedad o preocupación excesiva de sufrir o repetir una crisis y no tanto a separarse de la figuras de apego. En la fobia social el temor se centra en sentirse expuesto ante los demás. El rechazo escolar o la negativa de asistir al colegio puede formar parte del TAS y muchas veces encubre a éste último ${ }^{10}$. Se debe estar alerta ante el rechazo del niño de asistir a clases ya que esta situación puede deberse a una condición escolar abusiva (bulling) que puede estimular ansiedad evitativa y angustia a la separación del hogar. 
Tabla 2. Comparación clínica de la ansiedad por separación vs trastorno de ansiedad por separación

\begin{tabular}{|c|c|c|}
\hline Variable & Ansiedad por separación & Trastorno de ansiedad por separación \\
\hline $\begin{array}{l}\text { Edad de } \\
\text { aparición }\end{array}$ & Entre los 6 meses y 5-6 años & $\begin{array}{l}\text { Entre los } 3 \text { y } 18 \text { años (no es absoluto) } \\
\text { Preferentemente después de los } 6 \text { años }\end{array}$ \\
\hline $\begin{array}{l}\text { Desarrollo } \\
\text { evolutivo }\end{array}$ & $\begin{array}{l}\text { Ansiedad acorde al nivel de desarrollo mental } \\
\text { Ansiedad que permite al niño desarrollarse } \\
\text { Tiene carácter adaptativo }\end{array}$ & $\begin{array}{l}\text { Ansiedad desproporcionada al nivel de desarrollo mental } \\
\text { Ansiedad que retrasa el desarrollo emocional del niño } \\
\text { Es desadaptativa }\end{array}$ \\
\hline $\begin{array}{l}\text { Intensidad de } \\
\text { la ansiedad }\end{array}$ & $\begin{array}{l}\text { La expresión de la ansiedad a la separación de } \\
\text { la figura de apego es de intensidad similar a la } \\
\text { ansiedad que ocurre en otras situaciones } \\
\text { La ansiedad anticipatoria es de baja intensidad } \\
\text { y mejor regulada }\end{array}$ & $\begin{array}{l}\text { La expresión de la ansiedad a la separación de la } \\
\text { figura vinculada es de gran intensidad y mayor a la } \\
\text { ansiedad expresada situaciones habituales } \\
\text { Intensa ansiedad anticipatoria y preocupaciones } \\
\text { excesivas al alejamiento de la figura de apego }\end{array}$ \\
\hline Pensamiento & $\begin{array}{l}\text { Las ideas de daño o muerte en relación a las } \\
\text { figuras de apego son menos intensas y más } \\
\text { tolerables }\end{array}$ & $\begin{array}{l}\text { Las ideas de daño o muerte a las figuras de apego son } \\
\text { relevantes, perturbadoras y con respuestas catastróficas }\end{array}$ \\
\hline Temperamento & $\begin{array}{l}\text { El egocentrismo y las relaciones empáticas son } \\
\text { mejor moduladas } \\
\text { Autoestima bien regulada }\end{array}$ & $\begin{array}{l}\text { Egocentrismo marcado, requiriendo constantemente la } \\
\text { atención de los padres } \\
\text { Tendencia a la baja autoestima }\end{array}$ \\
\hline $\begin{array}{l}\text { Estilos de apego } \\
\text { observado }\end{array}$ & $\begin{array}{l}\text { Estilo de apego seguro } \\
\text { Vinculación sincrónica y armónica }\end{array}$ & $\begin{array}{l}\text { Estilo de apego inseguro } \\
\text { Vinculación asincrónica y disarmónica }\end{array}$ \\
\hline $\begin{array}{l}\text { Reacción de la } \\
\text { díada a la } \\
\text { separación }\end{array}$ & $\begin{array}{l}\text { En la observación clínica, la díada madre-hijo } \\
\text { se aprecia frecuentemente armónica y tranquila } \\
\text { frente a la separación }\end{array}$ & $\begin{array}{l}\text { En la observación clínica la díada madre-hijo en su } \\
\text { conjunto se aprecia tensionada y sobrereactiva a } \\
\text { situaciones de separación. }\end{array}$ \\
\hline $\begin{array}{l}\text { Padres y } \\
\text { familia }\end{array}$ & $\begin{array}{l}\text { Familias por lo general funcionales } \\
\text { Padres contenedores } \\
\text { Frecuentemente ausencia de psicopatología } \\
\text { ansiosa en la familia }\end{array}$ & $\begin{array}{l}\text { Familias con tendencia a ser aglutinadas } \\
\text { Padres generalmente sobreprotectores o inseguros } \\
\text { Probable psicopatología ansiosa en otros miembros } \\
\text { familiares }\end{array}$ \\
\hline Eventos vitales & Eventos de vida negativos infrecuentes & $\begin{array}{l}\text { Presencia de eventos de vida negativos (experiencias o } \\
\text { riesgos de separaciones traumáticas tempranas) }\end{array}$ \\
\hline $\begin{array}{l}\text { Efectos } \\
\text { psicosociales }\end{array}$ & $\begin{array}{l}\text { La ansiedad no interfiere en el funcionamiento } \\
\text { del niño aunque lo puede tensionar o inquietar } \\
\text { Sin deterioro en áreas importantes (colegio, } \\
\text { relaciones sociales) }\end{array}$ & $\begin{array}{l}\text { La ansiedad interfiere en el funcionamiento del niño y } \\
\text { causa deterioro en al menos un área importante de su } \\
\text { vida (colegio, relaciones sociales) }\end{array}$ \\
\hline Escolaridad & $\begin{array}{l}\text { El rechazo escolar está ausente, y si lo hay es } \\
\text { transitorio }\end{array}$ & Rechazo escolar evidente y muchas veces infranqueable \\
\hline Comorbilidad & Rara asociación a trastornos psiquiátricos & $\begin{array}{l}\text { Con frecuencia se asocia comorbilidad psiquiátrica (otros } \\
\text { trastornos de ansiedad y/o depresión) }\end{array}$ \\
\hline Pronóstico & $\begin{array}{l}\text { Tendencia a la regresión y remisión espontánea } \\
\text { La resolución ayuda a la autonomía emocional } \\
\text { y a las relaciones interpersonales seguras }\end{array}$ & $\begin{array}{l}\text { El trastorno se inicia en la infancia y puede prolongarse } \\
\text { hacia la adultez, pudiendo asociarse posteriormente a } \\
\text { psicopatología de tipo ansioso o de ánimo depresivo }\end{array}$ \\
\hline
\end{tabular}

\section{Etiología del TAS}

La interrelación de factores provenientes del temperamento, la neurobiología, el vínculo, el estilo parental y familiar es considerada en el desarrollo del TAS.

\section{a. Temperamento}

Se ha observado que los niños inhibidos y temerosos presentan un sistema autonómico con el tono simpático aumentado por lo que tendrían un mayor riesgo de desarrollar patología ansio$\mathrm{sa}^{12}$, y un mayor riesgo de presentar trastornos ansiosos no sólo en la infancia sino que también en la adolescencia ${ }^{13}$. Se cree que el temperamento y el comportamiento inhibido, tienen una importante base genética ${ }^{14}$. Estudios en gemelos evidencian que tanto las influencias genéticas como ambientales son importantes en el surgi- 
miento del trastorno de ansiedad por separación. La influencia genética es mayor en las niñas que en niños y a mayor edad de estos; por lo tanto, la influencia ambiental en el desarrollo de los cuadros ansiosos sería mayor a menor edad del niño y más importante en los varones ${ }^{10}$.

\section{b. Apego y regulación de la ansiedad}

La seguridad emocional es el principal objetivo del apego o vínculo afectivo. El apego es toda conducta por la cual un individuo mantiene o busca proximidad con otra persona considerada más fuerte y segura ${ }^{15}$. La conducta de apego permite utilizar al cuidador principal como base segura, desde la cual se explora lo desconocido. Desde la perspectiva de la teoría del apego, el ser humano no nace con la capacidad de regular sólo sus reacciones emocionales, sino que necesita un sistema regulador que es el vínculo. El apego seguro se caracteriza por adecuada manifestación de la ansiedad frente a la separación y adecuado re-aseguramiento al volver a encontrarse con la madre o figura vinculada. El apego ansioso/evitativo demuestra distanciamiento emocional durante la separación y desinterés en el reencuentro con la madre. En el apego ansioso/resistente el niño muestra ansiedad a la separación pero no se tranquiliza al reunirse con su madre, es perturbado por la separación y tienen dificultad en reponerse. La organización vincular segura guarda relación con la sensibilidad y sintonía de la madre a las señales del niño, mientras que la insegura se relaciona con excesiva ansiedad o inseguridad materna ${ }^{15}$. Los niños con vínculo inseguro tienen mayor probabilidad de sufrir trastornos de ansiedad en la infancia y adolescencia al compararlos con niños con vínculos seguros $^{16}$. La situación de separación o desapego transitorio en una díada vinculada de manera insegura, es vivida en la relación madre-hijo con gran tensión, con repercusión en lo emocional y en lo cognitivo ${ }^{2}$. Desde la perspectiva de la teoría del vínculo, se debe considerar que tanto el niño como la madre pueden ser agentes sintomáticos, es decir "responsables" de la manifestación del cuadro clínico. Desde este punto de vista es necesario ante una angustia excesiva en el niño, evaluar el funcionamiento de la relación diádica madre-hijo.

\section{c. El sistema familiar, la ansiedad parental $y$ el estilo de crianza}

Las familias de tipo aglutinada pueden ser fuente de excesiva ansiedad por separación, ya que en ellas se altera el desarrollo de la autonomía emocional por la existencia de vínculos demasiados estrechos que entorpecen la diferenciación emocional. En estas familias los padres se caracterizan por un estilo ansioso y sobreprotector y el grupo familiar tienden a la evitación del conflicto, por lo tanto, la ansiedad tiende a expresarse como somatizaciones ${ }^{17}$. Se ha observado que los hijos de padres que presentan trastornos de ansiedad tienen mayor riesgo de desarrollar trastornos ansiosos ${ }^{18}$. El TAS es más común en niños cuyos padres tienen antecedentes de trastornos de ansiedad o trastorno depresivos ${ }^{19}$. El estilo de crianza ansioso y el control parental están relacionados significativamente a TAS en niños escolares ${ }^{20}$. El control excesivo y la sobreprotección de los padres pueden significar en el niño que éste crea que el mundo sea ante todo un lugar peligroso. Este temor injustificado interfiere las capacidades del niño ${ }^{21}$.

\section{d. Hallazgos neurobiológicos}

Todo trastorno ansioso puede suponer la activación de la angustia con sus componentes somáticos, psíquicos y conductuales, mediados por la respuesta al estrés ${ }^{2}$. El estrés es la respuesta del organismo a estímulos procesados como peligrosos, activándose el sistema simpático, liberando cortisol la glándula adre$\mathrm{nal}^{22}$. La amígdala cerebral, como mediador de la actividad autonómica, se ha asociado al apego humano inseguro ${ }^{23}$. En el sistema neuroquímico responsable de la angustia, están involucrados al menos 4 sistemas de neurotransmisores: el serotoninérgico, gabaérgico, dopaminérgico y noradrenérgico ${ }^{2}$. Algunos estudios han relacionado la desregulación en el sistema noradrenérgico al desarrollo de ansiedad excesi$\mathrm{va}^{24}$. Un funcionamiento alterado del eje hipotálamo-hipófisis-suprarrenal ha sido relacionado con altos niveles de ansiedad en niños y adolescentes, y síntomas de ansiedad por separación podrían predecir un nivel de concentración elevada de cortisol ${ }^{25}$. 


\section{Tratamiento}

Es conveniente al tratar a un niño con TAS integrar intervenciones psicosociales y farmacológicas. La psicoterapia cognitivo conductual y los farmacoterapia han demostrado ser los tratamientos más efectivos en el tratamiento de la ansiedad excesiva del TAS ${ }^{26-28}$. Cuando el TAS es leve, se debe iniciar el tratamiento con psicoterapia y añadir fármacos en caso necesario. Si el cuadro clínico es más severo, conviene iniciar terapia conjunta.

\section{a. Psicoterapia}

Estudios controlados han documentado la efectividad de la Terapia Cognitivo Conductual (TCC) en el tratamiento del TAS y otros trastornos de ansiedad en la infancia y adolescen$\mathrm{cia}^{29}$. La TCC puede ser individual, grupal y con modalidad que involucre a los padres o cuidadores afectivos del niño. Las principales intervenciones terapéuticas conductuales son: educación afectiva, uso de técnicas cognitivas, intervenciones con exposición gradual con refuerzos positivos y técnica de relajación. La educación afectiva se refiere a que el niño ansioso aprenda a identificar y a comprender mejor los signos fisiológicos, conductuales y cognitivos que forman parte de la ansiedad como emoción. Este modelo tripartito de la emoción le enseña al niño a distinguir entre sentimientos excesivos y poco realistas de sentimientos armónicos y funcionales. En el proceso terapéutico el niño aprende un automonitoreo de sus sensaciones, pensamientos y respuestas conductuales, de modo de aprender estrategias de enfrentamiento a la angustia que le sean más funcionales y adaptativas. La exposición gradual consiste en que el niño paulatinamente y de manera protegida enfrenta situaciones temidas por él. Este proceso considera la habituación, principio formativo que postula la declinación natural de la ansiedad asociada a situaciones temidas, si el niño las enfrenta frecuentemente por períodos prolongados de tiempo. Para un mejor resultado debe contarse con la cooperación de una persona que contenga y tranquilice al niño durante la exposición a lo temido. El refuerzo positivo o premio, se debe utilizar para alentar el esfuerzo del niño al cambio conductual y complementa las otras técnicas del $\mathrm{TCC}^{10}$. La participación de los padres en la terapia de los trastornos de ansiedad, mejora la reducción de los síntomas ansiosos al compararlas con terapias conductuales en que los padres no participan activamente ${ }^{30}$.

\section{b. Farmacoterapia}

La farmacoterapia debería ser considerada en el tratamiento de los trastornos de ansiedad si la sintomatología es severa o invalida notoriamente el funcionamiento del niño, o en el caso de que de 6-8 semanas de psicoterapia (considerando la intervención con los padres), no logró reducir los síntomas ansiosos en el niño. Los fármacos de primera elección en el tratamiento del TAS son los inhibidores selectivos de recaptación de serotoninas (ISRS) ${ }^{31}$. Los ISRS han demostrado su efectividad en el tratamiento de los trastornos de ansiedad y los trastornos del ánimo por su efecto ansiolítico y antidepresivo. Estudios controlados demuestran la eficacia y la seguridad de los ISRS en niños y adolescentes con trastornos de ansiedad ${ }^{31,32}$. Dentro de los ISRS se ha comprobado la eficacia de la fluoxetina en el tratamiento con niños, algunos pacientes que no han respondido a intervenciones terapéuticas han presentado mejoría sintomática al uso de este fármaco ${ }^{33,34}$. Los efectos colaterales de la fluoxetina son sido leves y breves ${ }^{35}$. La sertralina y fluvoxamina, también fármacos ISRS, han demostrado ser más eficaces que placebo en la disminución de síntomas ansiosos. Esta reducción sintomática se ha asociado a mejoría clínica glo$\mathrm{bal}^{32,36}$. Una segunda línea de farmacoterapia pueden ser las benzodiacepinas, tales como el alprazolam y el clonazepam. No hay suficiente datos empíricos que demuestren la eficacia del uso de benzodiacepinas en el tratamiento de los trastornos ansiosos en niños y adolescentes. Ensayos clínicos controlados no se ha observado diferencia significativa entre uso de benzodiacepinas versus placebo en pacientes con trastornos ansiosos ${ }^{31,37,38}$. Estos medicamentos con efecto ansiolítico más inmediato, podrían ser utilizados en conjunto con los ISRS mientras se espera que comience el efecto de estos últimos (1 a 2 semanas). Por ser moléculas de vida 
media corta las benzodiacepinas pueden ser utilizadas en la mañana ya que reducen rápidamente la ansiedad, por tanto ayudan al paciente a tolerar la partida al colegio cuando el rechazo escolar acompaña a la ansiedad por separación. Debido a sus efectos adversos, como su potencial de dependencia, las benzodiacepinas deben ser indicadas por períodos cortos y con estricta supervisión. Actualmente los antidepresivos tricíclicos como la imipramina, son de escasa utilización debido a sus efectos adversos y su eficacia no demostrada en comparación a placebo.

La duración del tratamiento farmacológico para el TAS no está bien establecida pero debería ser lo suficiente para que la sintomatología del paciente se haya reducido, y el entrenamiento en psicoterapia ya le permita ser capaz de modular su ansiedad sin la necesidad del uso de fármacos. Al suspender la medicación debe monitorearse una eventual recurrencia de la ansiedad excesiva.

\section{Discusión}

La AS es esperable en el niño como parte de su desarrollo emocional surgiendo desde los 6 meses hasta los 3 años, pudiendo extenderse en regresión progresiva hasta los inicio de la etapa escolar. Las reacciones de AS del desarrollo pueden ser vistas como señales discretas del proceso vinculación-separación. A medida que el niño progresivamente crece con un vínculo emocional seguro, será capaz de tolerar separaciones de su madre cada vez más largas. Este desarrollo es fruto de interacciones y de experiencias vividas entre el niño, su madre (o su figura de apego) y el entorno. ¿De qué manera el niño logra mantener una imagen mental de su madre, incluso en ausencia de ella y no sentirse solo? El niño podrá tener conductas cada vez más independientes cuando sea capaz de sostener una imagen mental estable de su madre o de la persona con la cuál esté vinculado y esto se da porque a nivel cognitivo se pone en marcha, hacia los 18 meses de edad, la permanencia objetal o representación mental del objeto ${ }^{39}$. Esta función mental permite que el niño sea capaz de imaginar una "mamá" sin tener una "mamá visible", asumiendo progresivamente que ella puede no estar cerca o visible pero se halla en el mundo. Esta experiencia cognitiva y emocional es vivida por el niño con reacciones ansiosas de búsqueda. El tiempo que el niño puede mantener una representación interna de su madre cuando esta no está crece progresivamente según su edad. A medida que esto ocurre, el niño alcanza niveles mayores de autonomía. En consecuencia, podría afirmarse que la AS del desarrollo juega un rol de ajuste emocional al proceso de socialización normal del niño.

El TAS, a diferencia de la AS es un proceso de carácter psicopatológico cuya sintomatología es desadaptativa y que tiene repercusión en el funcionamiento social y/o escolar del niño. Diferenciar estas dos expresiones ansiosas en la evaluación clínica del niño es primordial ya que cada condición tiene un modo distinto de intervención. Los siguientes criterios pueden ser considerados por el pediatra ante un niño excesivamente ansioso y que orientan a un TAS:

a. Intensidad del llanto: Ante una separación las manifestaciones de ansiedad son vividas por el niño con intenso llanto que puede acompañarse de rabietas que puede durar horas y que se calman sólo con la presencia de la madre (o la figura de apego), si no se han calmado antes por agotamiento físico.

b. Repetición: Las manifestaciones de reacciones ansiosas intensas se repiten ante cada separación o alejamiento de la madre.

c. Desorganización conductual: Alejado de la madre, el niño no logra organizar sus actividades incluidas las de juego. Le falta sosiego emocional o bien se ve abatido, temeroso y no logra tener calma.

d. Anticipación ansiosa activa: El niño (y también la madre) intuye que puede quedar separado de su madre y comienza a aferrarse a ella o a llorar. Se inicia un estrechamiento de la relación madre-hijo con alta resistencia de ambos a alejarse. El lenguaje verbal en estas manifestaciones pierde relevancia. Al poner atención a los gestos observamos como estos alcanzan alta escenificación e intensidad.

e. Anticipación ansiosa pasiva: La madre del niño (en algunos casos el mismo niño) nos puede relatar que su hijo tiene miedos perma- 
nentes, pesadillas, o temor a sufrir accidentes ante la posibilidad de separarse de ella o que manifiesta temor persistente de no volver a verla o de que ella sufra un accidente. Un tema recurrente en el niño es su miedo a quedarse solo.

Pueden darse las siguientes situaciones en el modo de presentación de un TAS al considerar la edad del niño como factor de orden cronológico:

- Continuidad sintomática: El TAS emerge en el contexto de una AS normativa del desarrollo que no se ha mitigado y se ha prolongado más allá de lo esperado. El niño "ya está grande" para reaccionar y sigue reaccionando con ansiedad y temor a las separaciones a pesar de su edad más avanzada.

- Resurgimiento sintomático: Las manifestaciones del TAS emergen a una edad en que las reacciones a la separación ya no deberían surgir porque el niño ya logró modular su ansiedad de separación con anterioridad. Existiría un período de normalización de la ansiedad previo.

En términos teóricos conviene considerar la existencia de una continuidad dimensional entre la AS del desarrollo y el TAS, en la cuál este último alcanza significación psicopatológica en función de la intensidad y del contexto de sus manifestaciones.

La intervención terapéutica que se amerite en el caso de la AS del desarrollo será de psicoeducación a los padres y a nuestros pacientes, a modo de prevenir la ansiedad por separación de carácter desadaptativo (anexo 1). Estas medidas las pueden poner en práctica los padres especialmente en situaciones en las que el niño está aprendiendo a separarse por motivos de socialización normativa, como es el caso del ingreso al sistema escolar o cuando el niño participa en actividades recreativas fuera de su hogar. Conviene que el pediatra en el control del niño sano evalúe la evolución de la AS con la información que la madre proporciona respecto de como su hijo reacciona a las separaciones, y ponga atención a las reacciones que el niño tiene cuando es atendido y examinado, observe como el niño se relaciona y cuanto tolera estar distante de su madre. Frente a exacerbaciones o sobrereacciones ansiosas del niño convendrá reevaluar su situación de un modo más focalizado para confirmar o descartar un TAS.

El tratamiento del TAS, en opinión de los autores, requiere de la participación de un especialista en salud mental de niños. El tratamiento debe adaptarse a cada caso en particular y la información aportada por el pediatra o el médico familiar de las características del niño y de su funcionamiento global previo, será de gran ayuda para el especialista. El trabajo conjunto entre el pediatra, psicólogo y psiquiatra permite integrar las distintas modalidades terapéuticas con sus respectivos abordajes: manejo conductual y del estilo de vinculación de la madre y su hijo, apoyo familiar y terapia farmacológica. A nuestro parecer, el pediatra continúa cumpliendo un importante rol en el equipo tratante, especialmente cuando el especialista necesita dilucidar o dimensionar las quejas físicas que son muy frecuentes en los pacientes con TAS. Agreguemos que el pediatra es quién posteriormente mantendrá contacto más cercano con el niño y sus padres y observará la evolución del desarrollo emocional. En caso de ausencia de un especialista a quién derivar o tardanza de la interconsulta, el pediatra debería iniciar un abordaje terapéutico. Para este fin sugerimos que tenga en consideración lo siguientes: que el TAS puede considerarse como un estado de dependencia mutua excesiva entre la madre y su hijo y no sólo como un trastorno individual del niño. Esto significa que la intervención o indicaciones que daremos si bien van orientadas al niño, deben siempre tomar en cuenta la reacción de la madre ya que de esta reacción puede depender el éxito de una prescripción. Una madre temerosa o insegura puede ser resistente a los cambios y a las indicaciones, especialmente al uso de fármacos. Otro punto es considerar el restablecimiento de la funcionalidad del niño lo antes posible. El retorno al colegio de manera gradual y supervisada puede ser un foco de intervención (tener presente el punto anterior). También la detección de posibles dificultades que pueda estar pasando la familia y el rol que el niño juega en ellas, puede ser un foco de atención y consejería que el pediatra o médico familiar puede desarrollar logrando cambios que pueden aliviar la carga emocional en el niño. 
El tratamiento farmacológico puede iniciarlo el pediatra cuando la sintomatología lo amerite, pero para este fin debe estar en conocimiento de los efectos clínicos y adversos de los fármacos prescritos.

En toda intervención terapéutica de los trastornos ansiosos no debemos olvidar de transmitir tanto al niño como a sus padres un sentimiento de tranquilidad y comprensión ante la angustia que los aqueja y de seguridad que con tratamiento adecuado estos trastornos remiten.

\section{Referencias}

1.- Mayes L, Gillian W, Stout-Sosinsky L: The infant and toddler. En: Lewis's Child and Adolescent Psychiatry: A Comprehensive Textbook, $4^{\text {th }}$ Edition, Martin A, Volkmar F. (Eds), 2007.

2.- Leckman J, Vaccarino FM, Lombroso PJ: Development of the symptom of anxiety. En: Child and Adolescent Psychiatry: A Comprehensive Textbook ( $3^{\text {rd }}$ ed.) Lewis M (Ed.), Williams \& Wilkins, 2002.

3.- Gabbard G: Trastornos de Ansiedad. En: Psiquiatría Psicodinámica en la Práctica Clínica, $3^{\mathrm{a}}$ Ed. Médica Panamericana, 2006.

4.- Thurber C, Walton E; American Academy of Pediatrics Council on School Health: Preventing and Treating Homesickness. Pediatrics 2007; 119: 192-201.

5.- Freeman JB, García AM, Leonard HL: Anxiety disorders. En: Child and Adolescent Psychiatry: A Comprehensive Textbook ( $3^{\text {rd }}$ ed.) Lewis M (Ed.), Williams \& Wilkins, 2002.

6.- Compton SN, Nelson AH, March JS: Social phofia and separation anxiety symptoms in community and clinical samples of children and adolescent. J Am Acad Child Adolesc Psychiatry 2000; 39: 1040-6.

7.- McGee R, Feehan M, Williams S, Partridge F, Silva $P$, Kelly J: DSM III disorders in a large sample of adolescents. J Am Acad Child Adolesc Psychiatry 1990; 29: 611-9.

8.- Manicavasagar V, Silove D, Hadzi-Pavlovic D: Subpopulations of early separation anxiety: relevance to risk of adult anxiety disorders. J Affect Disord 1998; 48: $181-90$

9.- American Psychiatric Association: Manual Diagnóstico y Estadístico de los Trastornos Mentales IV (DSM IV). Ed. Masson, Barcelona 1995.

10.- Hanna G, Fischer DJ, Fluent TE: Separation Anxiety Disorder and School Refusal in Children and Adolescents. Pediatr Rev 2006; 27: 56-63.

11.- Strauss $C$, Lease $C$, Kazdin A, Dulcan M, Last $C$ : Mutimethod assessment of the social competence of anxiety disordered children. J Clinical Child Psychology 1989; 18: 184-90.

12.- O'Laughlin EM, Meeker EC, Bischoff LG: Predictors of children's emotional distress in a mother-absent situation: implications for caregiving research 2000; 161: 235-52.

13.- Kagan J, Snidman N: Early childhood predictors of adult anxiety disorders. Biol Psychiatry 1999; 46: 1536-41.

14.- Robinson JL, Kagan J, Reznick JS, Corley R: The heritability of inhibited and uninhibited behavior: a twin study. Dev Psychol 1992; 28: 1030-7.

15.- Bowlby J: La separación afectiva. Ed. Paidós Ibérica 1993.

16.- Warren SL, Huston L, Egeland B, Sroufe A: Child and Adolescent anxiety disorders and early attachment. J Am Acad Child Adolesc Psychiatry 1997; 36: 63744.

17.- Creswell C, Cartwright-Hatton S: Family treatment of child anxiety: outcomes, limitations and future directions. Clin Child Fam Psychol Rev 2007; 10: 232-52.

18.- Weissman MM, Leckman JE, Merikangas KR, Gammon $G D$, Prusoff $B A$ : Depression and anxiety disorders in parents and children: results from the Yale Family Study. Arch Gen Psychiatry 1984; 41: 845-52.

19.- Biederman J, Faraone SV, Hirshfeld-Becker DR, Friedman D, Robin JA, Rosembaun JF: Patterns of psychopathology and dysfunction in high-risk children of parents with panic disorders and major depression. Am J Psychiatry 2001; 158: 49-57.

20.- Muris P, Meesters C, Merckelbach H, Sermón A, Zwakhalen S: Worry in normal children. J Am Acad Child Adolesc Psychiatry 1998; 37: 703-10.

21.- Rapee RM: Potencial role of childrearing practices in the development of anxiety and depression. Clin Psychol Rev 1997; 17: 47-67.

22.- Heim C, Nemeroff CB: The role of childhood trauma in neurobiology of mood and anxiety disorders: preclinical and clinical studies. Biol Psychiatry 2001; 49: 1023-39.

23.- Lemche E, Giampietro VP, Surguladze SA, et al: Human attachment security is mediated by the amygdala: Evidence from combined fMRI and psychophysiological measures. Human Brain Mapp 2005; 27: 623-35.

24.- Sallee FR, Sethuraman G, Sine L, Liu H: Yohimbine challenge in children with anxiety disorders. Am J Psychiatry 2000; 157: 1236-42.

25.- Kallen VL, Tulen JH, Utens EM, Treffers PD, De Jong $F H$, Ferdinad RF: Association between HPA axis functioning and level of anxiety in children and adolescents with an anxiety disorders. Depress Anxiety 2008; 25: 131-41.

26.- Kendall $P C$ : Treating anxiety disorders in children: Results of a randomized clinical trial. J Consult Clin Psychol 1994; 62: 100-10.

27.- Barret PM, Duffy AL, Dadds MR, Rapee RM: Cognitive-behavioral treatment of anxiety disorders in children: long-term (6-years) follow-up. J Consult Clin Psychol 2001; 69: 135-41.

28.- Allen AJ, Leonard H, Swedo SE: Current knowledge of medications for the treatment of childhood anxiety disorders. J Am Acad Child Adolesc Psychiatry 1995; 
34: $976-86$

29.- Labellarte MJ, Ginsburg GS, Walkup JT: The treatment of anxiety disorders in children and adolescents. Biol Psychiatry 1999; 46: 1567-78.

30.- Barrett PM, Dadds MR, Rapee RM: Family treatment of chilhood anxiety: a controlled trial. J Consult Clin Psychol 1996; 69: 333-42.

31.- Dieleman GC, Ferdinand RF: Pharmacotherapy for social phobia, generalised anxiety disorder and separation anxiety disorder in children and adolescents: an overview Dutch Journal of Psychiatry 2008; 50: 43-53.

32.- Rynn MA, Sigueland L, Rickels K: Placebo-controlled trial of sertraline in the treatment of children with generalized anxiety disorder. Am J Psychiatry 2001; 158: 2008-14.

33.- Birmaher B, Waterman GS, Ryan N, et al: Fluoxetine for childhood anxiety disorders. J Am Acad Child Adolesc Psychiatry 1994; 33: 993-9.

34.- Clark DB, Birmaher B, Axelson DA, et al: Fluoxetine for the treatment of childhood anxiety disorders: openlabel, long-term extension to a controlled trial. J Am Acad Child Adolesc Psychiatry 2005; 44: 1263-70.

35.- Birmaher B, Axelson DA, Monk K, et al: Fluoxetine for the treatment of childhood anxiety disorders. J Am Acad Child Adolesc Psychiatry 2003; 42: 41523.

36.- Walkup JT, Labellarte MJ, Riddle MA, et al: Fluvoxamine for the Treatment of Anxiety Disorders in Children and Adolescents. N Engl J Med 2001; 344: 1279-85.

37.- Gaae F, Milner J, Rizzotto L, Klein RG: Clonazepam in childhood anxiety disorders. J Am Acad Child Adolesc Psychiatry 1994; 33: 372-6.

38.- Bernstein GA, Garfinkel BD, Borchardt CM: Comparative studies of pharmacotherapy for school refusal. J Am Acad Child Adolesc Psychiatry 1990; 29: 773-81.

39.- Papalia DE, Wendkos S, Duskin R: Psicología del desarrollo, 8 a edición. McGraw-Hill (Ed), 2001.

Anexo 1. Estrategias de prevención de la ansiedad excesiva. Guía para los padres para ser utilizadas con sus hijos en situaciones de separación

- Incluir al niño, en la medida de lo posible en la decisión de estar fuera de casa. Tomar parte incrementa la sensación de autocontrol. Contrariamente, si el niño es forzado a dejar la casa sin ser parte de la decisión, aumenta la intensidad de la ansiedad al separarse

- Si el niño estará fuera de casa le podría decir: "La mayoría de las personas extrañan su hogar cuando están lejos. Significa que extrañas y extrañar es normal. Hay muchas cosas que puedes pensar y hacer que te pueden ayudar cuando te sientas solo o temeroso"

- De instrucciones específicas al niño para manejar la ansiedad, esta acción puede fomentar la seguridad del niño durante el tiempo de separación

- Hacer algo entretenido, como jugar con amigos para olvidar la ansiedad por el hogar

- Hacer algo para sentirse más cercano al hogar (escribir una carta, mirar una foto, comunicarse).

- Hablar con alguien que lo pueda hacer sentir mejor y le dé seguridad

- Pensar que el tiempo fuera de casa será corto

Pensar en los que quieres e imagina que dirían ellos para ayudarte

- Conozca junto al niño el lugar donde estará, esto aumenta la familiaridad, reduciendo la angustia

- Conocer gente en el nuevo ambiente tranquiliza. Tener al menos una cara familiar hace que disminuya significativamente la ansiedad

- Abstenerse de expresar sentimientos ansiosos o ambivalentes respecto a la estadía del niño fuera de casa. Padres bien intencionados pueden exacerbar la ansiedad por separación con frases como: "espero que no me extrañes tanto", "espero que te encuentres bien y no pase nada malo" o "se grande, no nos extrañes". Dar a los niños algo de que preocuparse, aumenta la probabilidad de que tengan pensamientos ansiosos con respecto a la casa. Idealmente los padres deberían expresar entusiasmo y optimismo sobre la separación y el nuevo ambiente. Deberían ser compartir su propia ansiedad con entre ellos o con otros adultos, pero no con sus hijos

- Mantener una real perspectiva del tiempo, use un calendario para mostrarle al niño concretamente que el tiempo fuera de casa es limitado. Los calendarios son útiles para dar a los niños una perspectiva de la duración de su estadía fuera del hogar

Basado en: Preventing and Treating Homesickness (4) 